\title{
Pospolítica y educación en Chile (1990-2012): Fases y desfases del experimento neoliberal chileno en educación ${ }^{1}$
}

\author{
Pospolítica e Educação no Chile (19920-2012): fases e desfases \\ do experimento neoliberal chileno na educação
}

\section{Jorge Alarcón-Leiva² \\ Emma Johnston ${ }^{3}$ \\ Claudio Frites-Camilla ${ }^{4}$}

\section{Resumo}

O trabalho adota a ideia da transição política chilena como uma "democracia dos acordos", a que interpreta como uma forma de negação da política. A partir disto, são examinados alguns dos eventos da educação chilena, para compreender as limitações existentes neste contexto em função de uma adequada promoção do direito à educação.

Palavras-chave: Pospolítica; Democracia dos acordos; Consenso; Educação; Transição.

\section{Resumen}

El trabajo adopta la idea de la transición política chilena como una "democracia de los acuerdos", la que interpreta como una forma de negación de la política. A partir de ello, se examinan algunos de los eventos de la educación chilena, para comprender las limitaciones existentes en este contexto en función de una adecuada promoción del derecho a la educación.

Palabras clave: Pospolítica, Democracia de los acuerdos, Consenso, Educación, Transición.

\begin{abstract}
This article examines Chilean education policy. It uses the idea of the transition as a "democracy of agreements", which it interprets as the negation of politics. Using this framework, it analyses some of the key aspects of recent Chilean educational policy, in order to understand the limitations preventing the adequate promotion of the right to education.
\end{abstract}

Key Words: Post-politics, "Democracy of agreements", Consensus, Education, Transition.

1 El texto ha sido escrito en el marco del Proyecto CONICYT REDES $120015 \mathrm{U}$. de TALCA-IIDE-Chile /UFPR-NUPE-Brasil y algunos de sus elementos fueron discutidos en el Seminario "Propuestas de Nueva Institucionalidad de la Educación Pública Chilena", en Enero 2014, Santiago de Chile. Una primera versión de este texto fue expuesto como parte de un curso para estudiantes de Postgrado de Educación, en la Universidade Federal do Paraná, Curitiba, Brasil, en octubre de 2013.

2 Doctor en Filosofía, profesor e investigador Instituto de Investigación y Desarrollo Educacional (IIDE), Universidad de Talca. Dirección electrónica: joalarcon@utalca.cl

3 Licenciada en Letras, Cambridge University. Estudiante de Magister en Estudios Latinoamericanos, Cambdridge University. Correo electrónico: efc. johnston@gmail.com

4 Sociólogo y Magister en Políticas Públicas, U. de Chile. Correo electrónico: clfrites@gmail.com 


\section{Introducción}

La política educativa chilena posterior a la dictadura constituye, según toda evidencia, un caso único. En este sentido, las voces que surgen actualmente en el debate educativo lo hacen en continuidad con los efectos del golpe de Estado de 1973.

En efecto, en Chile el golpe de Estado establecería una división social entre "[El polo victimario, el que] hace de la violencia (bruta e institucional) un instrumento de fanatización del Orden que opera como molde disciplinario de una verdad obligada, [y el polo victimado, el que] aprende traumáticamente a disputarle sentidos al habla oficial" (RICHARD, 1994, p. 55).

Tal división estaría en la base del proceso de transición a la democracia. De forma que cerrar el proceso de la transición se transformó en equivalente a eliminar la división social establecida tras el golpe de Estado. Lamentablemente, ello no fue posible y condicionó el carácter mismo de dicha transición: "pactada" y regida por "enclaves autoritarios" (GODOY, 1999; GARRETÓN y GARRETÓN, 2010).

\section{Consenso y olvido}

En esta sección se busca caracterizar la categoría de "posdemocracia" y la manera en que ésta se manifiesta en el Chile actual. En primer lugar, conviene señalar que, siguiendo a Rancière, la política está estrechamente ligada a la división de lo sensible, o sea a la "repartición de espacios y de tiempos, de lo visible y de lo invisible, del ruido y del lenguaje" (RANCIÈRE, 2005: 15). En este sentido, a la esencia de la política corresponde la tensión entre el cuerpo social ordenado y estructurado, donde cada parte tiene su lugar, y la parte de los sin parte, quienes desajustan ese orden exigiendo su derecho a voz (ŽIŽEK, 2008: 26).

Rancière llama a este proceso "subjetivación", lo que consiste en "reconfigurar la división de lo sensible, en introducir sujetos y objetos nuevos, en hacer visible aquella [parte] que no lo era" (RANCIÈRE, 2005: 15). El momento político por antonomasia tiene lugar cuando un nuevo interlocutor, una nueva parte de "los sin parte", logra irrumpir en el espacio de la hegemonía, alterando la configuración de las partes de la sociedad, cuestión que a la luz del debate educativo chileno, se daría a partir de las masivas movilizaciones del año 2006, que para Burton (2012) correspondería al "momento deliberativo" en la política educativa chilena.

A pesar de ello o quizás justamente por esto, desde Platón los filósofos políticos se han ocupado de negar este momento para controlar mejor el demos y la distribución de sus partes (RANCIÈRE, 1996: 86). Tales negaciones pueden adoptar variadas formas, entre las que destacan las "grandes figuras de la filosofía política": la arquipolítica, la parapolítica y la metapolítica (RANCIÈRE, 1996; ŽlŽEK, 2008).

La arquipolítica, cuyo modelo propone precisamente Platón, se refiere a la distribución de cuerpos en funciones sociales de acuerdo con su naturaleza: una "comunidad concebida como cuerpo animado por el alma una del todo" (RANCIÈRE, 1996: 92), la que controla totalmente las maneras de ser y las maneras de pensar para que no existan "resquicios desde los que pueda emerger el momento/acontecimiento político" (ŽIŽEK, 2008: 28).

La segunda figura, la parapolítica, originalmente fue propuesta por Aristóteles y ha sido el modelo político preponderante en las sociedades occidentales desde la Revolución Francesa. En ella se acepta el conflicto político, siempre y cuando se identifique con "el conflicto por la ocupación de los "puestos de mando»" (RANCIĖRE, 1996: 96). Dicho de otra manera, el conflicto político se reduce a la competencia/alternancia entre partidos políticos $u$ otros actores legitimados o reconocidos como partes del cuerpo político.

Por su parte, si bien la tercera figura, la metapolítica marxista, reconoce la existencia del conflicto, le resta importancia, ya que considera que "lo social y las clases sociales" son "el movimiento real de la sociedad" (RANCIĖRE, 1996: 107), por lo que el conflicto en el campo político no es más que un reflejo del conflicto perteneciente a los ámbitos económicos y sociales. Žižek hace referencia a una cuarta categoría de negación 
(que Rancière no menciona), la ultrapolítica, en que se intenta despolitizar el conflicto, militarizándolo, convirtiendo la política en una guerra entre un Nosotros y el Enemigo (ŽlŽEK, 2008: 29).

La preocupación por delimitar con precisión la esfera política, con el fin de controlar mejor la distribución de las partes, ha sido un constante en la filosofía política desde sus orígenes. Sin embargo, en los últimos cuarenta años, se han producido numerosos cambios en el escenario mundial, los cuales a su vez han ocasionado cambios en la experiencia de la ciudadanía, con el resultado de que los antiguos modelos políticos ya no parecen adecuados para analizar la contingencia política. Entre estos cambios se destacan el derrumbe de las ideologías y el debilitamiento de los ideales colectivos tras el fin de la Guerra Fría, además de la mayor importancia dada a la economía como fuerza motor de la sociedad y de la política misma, en un sentido diverso a aquél ya esbozado en el modelo marxista de negación del momento político.

En este mundo desideologizado, donde "el modo en que funciona la economía [...] se acepta como una simple imposición del estado objetivo de las cosas", las buenas ideas ya no tienen relación alguna con la ideología, sino que son "las que funcionan" (ŽlŽEK, 2008: 110). Todo eso va a la par con lo que Lipovetsky ha denominado "la época del posdeber", en que el individualismo prima sobre los ideales colectivos y el deber hacia la comunidad, por lo que los individuos "están poco inclinados al bien público" (LIPOVETSKY, 1993: 203).

Más específicamente, en el contexto chileno, la pérdida de las grandes ideologías y los ideales colectivos se hizo evidente en la manera en que los gobiernos de la Concertación de Partidos por la Democracia abordaron la transición política. La transición adoptaría el consenso como el momento político fundamental, aquél al que debía dirigirse toda acción política. Un momento en que el conflicto político es dialécticamente superado por la "democracia de los acuerdos"; lo que Moulián (1997: 37) Ilama, en clave hegeliana, "la etapa superior del olvido".

En el mismo sentido, Moulián argumenta que "la llamada transición ha operado como un sistema de trueques: la estabilidad, se dijo, tiene que ser comprada por el silencio" (1997: 32-33). La metáfora de ese silencio es el iceberg que formaba parte de la exposición chilena en la Exposición Universal de Sevilla, en el año 1992. El iceberg, que provenía del territorio antártico chileno, "representaba el estreno en sociedad del Chile Nuevo; limpiado, sanitizado" (MOULIÁN, 1997: 35).

Con todo, el consenso no es solo la "voluntad del olvido". Si la política está estrechamente ligada a la división de lo sensible y al proceso de subjetivación -a través del cual una parte de los sin parte logra irrumpir en el escenario político para conseguir hacerse oír-, el consenso se da cuando lo sensorial es unívoco, cuando una única interpretación se ha impuesto. Rancière (1996) caracteriza los contextos en los que el consenso está generalizado y legitimado por el régimen político vigente como ejemplos de posdemocracia: en Chile darían cuenta de ello no solo la educación, sino el sistema de salud y de pensiones. En estos contextos el régimen de lo privado se impuso al régimen de lo público, efectivamente (KRIPPNER y ALVAREZ, 2007; ATRIA, 2007), como la forma única de acuerdo con la cual la realidad social se hizo perceptible.

Así, la "interpretación correcta del mundo" correspondería al resultado de comprender los mecanismos de mercado que constituirían la verdadera arquitectura del orden social, sus principios elementales. Cabría decir aquí: la economía se transformó en la metafísica de la realidad social y la adscripción a su narrativa cuenta como el rito de entrada a un mundo regulado por relaciones de intercambio, no de sentido. De forma que esa narrativa se transformaría en el discurso público oficial; "público" solo en cuanto vela por el interés privado. A diferencia de la democracia, la posdemocracia es "una democracia que liquidó la apariencia, la cuenta errónea y el litigio del pueblo" y que es "reductible [...] al mero juego de los dispositivos estatales y las armonizaciones de energías e intereses sociales", crea y mantiene una ilusión de unanimidad, donde se ignoran las voces opositoras (RANCIÈRE, 1996: 129): "[S]ubraya la necesidad de abandonar las viejas divisiones ideológicas y de resolver las nuevas problemáticas con ayuda de la necesaria competencia del experto y deliberando libremente tomando en cuenta las peticiones y exigencias puntuales de la gente" (2008:32). Uno de los objetivos de la 
posdemocracia es lograr la estabilidad y la paz social tras "hacer desaparecer la apariencia perturbada y perturbadora del pueblo y su cuenta siempre falsa, detrás de procedimientos de presentificación exhaustiva del pueblo y sus partes y de armonización de la cuenta, de las partes y la imagen del todo" (RANCIÈRE, 1996: 130).

Por lo tanto, se puede sostener que Chile actual experimenta un estado de posdemocracia, motivada por la necesidad de reproducir el modelo económico y social de la dictadura, donde la política ya no se entiende como una lucha de alternativas sino que "existe sólo como historia, de las pequeñas variaciones, ajustes, cambios en aspectos, que no comprometan la dinámica global", lo que implica "la fagocitación del Nosotros por el Ellos" (MOULIÁN, 1997: 39), o sea, la desaparición de los sin parte.

Es decir, intentando superar las viejas divisiones ideológicas, buscando soluciones en el refulgente saber de los expertos, en las obras concretas que hacen enmudecer la retórica política y sumergiéndose en los deseos de la gente, el sistema se dividiría entre los integrados al sistema y los apocalípticos que se excluyen de él (HOPENHAYN, 1994). O, siguiendo a Žižek, entre tontos y pícaros.

\section{El pacto acosado}

La política nacional chilena venía siendo un proceso fluido y sin grandes roces, o con roces limados por la capacidad del consenso para neutralizar las discrepancias, hasta tanto algunos sucesos sociales de gran alcance -en 2006 y 2011, ambos ligados a la crisis de la educación en el país- produjeron anomalías para las cuales el sistema político institucional no ha mostrado aún capacidad de respuesta (CONSEJO, 2006; PANEL DE EXPERTOS, 2011; BELLEI, CONTRERAS \& VALENZUELA, 2010). Esta situación exige pensar en cómo pudo darse este fenómeno en el contexto de un país pacificado por la transición, adormecido por el consenso. La sección se propone justamente ofrecer una interpretación de los hechos relacionados con la política educativa nacional, desde la perspectiva esbozada previamente a propósito del "momento político".

Desde la perspectiva de la política educacional chilena, como una herramienta heurística se puede pensar que, como ha propuesto Burton (2012), el proceso de transición política abarca tres periodos claramente delimitados: desde 1990 a 2005, 2006 y desde 2006 hasta el momento en que se escribe este trabajo.

Al primero de tales periodos, Burton lo denomina de "representación limitada" y se caracteriza por la operación sin contrapeso del paradigma hegemónico y la consecuente debilidad de los movimientos sociales, lo que se enmarca dentro del modelo político de la posdemocracia, donde existe "una adecuación total entre las formas del Estado y el estado de las relaciones sociales” (RANCIÈRE, 1996: 129).

Se trata de los primeros años de democracia, aquellos en que colisionan lo que Corvalán llama la "narrativa centro estatista", previa a 1973, y la "narrativa de mercado". Esta última es la que introduce las importantes transformaciones en los años 80 y la que da lugar a la "economía neoliberal como la principal e incluso única disciplina [científica] capaz de generar lenguaje explicativo y analítico del sistema educativo" (CORVALÁN, 2013:68). De ese modo, la conformación de la agenda de política educativa corresponde al proceso de síntesis y construcción intelectual: un tiempo de consenso entre actores y policy makers que llevaría a las políticas de la Concertación a ser una "prolongación maquillada de aquellas de inspiración neoliberal del gobierno militar" (PICAZO, 2010: 64)

De manera que tales políticas concertacionistas, no lograrían modificar sustancialmente el diseño institucional del sistema educacional chileno instaurado por la dictadura, a saber: descentralización administrativa de la educación pública, financiamiento público para instituciones privadas (ALMONACID, 2004) e instalación de un cuasi-mercado en educación, establecido bajo el supuesto que las solas fuerzas del mercado lograrían mejorar el sistema en eficiencia y calidad (MIZALA y ROMAGUERA, 2005; Almonacid, 2008). Durante estos primeros años, las que luego aparecerían como evidentes "fallas de mercado" -problemas de agencia e incentivos y ausencia de accountability - se intentarían neutralizar mediante la introducción de medidas de 
mejora en el sistema y mediante el incremento de insumos educativos (MIZALA y ROMAGUERA, 2005). Es decir, más de lo mismo.

Por obra del gobierno de la Concertación de Partidos por la Democracia, en este periodo se desarrollan, asimismo, políticas de financiamiento que implicarían un fuerte aumento en el gasto, además de modificarse la situación de la profesión docente a través del Estatuto Docente, que favorecería mejoras laborales asociadas a la mayor estabilidad del empleo, entre otras cosas. Así también se implementa una serie de programas de mejoramiento, el nuevo marco curricular, la extensión de la jornada escolar, se modifica la naturaleza de contextos y procesos educativos, y se construyen consensos respecto a la necesidad y características de cambios en el sector (MIZALA y ROMAGUERA, 2005).

Como certeramente se ha indicado, subyace a estas transformaciones la pretensión de pasar del estado maximalista de los años 70 y del minimalista de los 80, a un "Estado capaz" (COX, 1997). Vale decir, a un Estado que ejerciera el margen de acción permitido por el diseño institucional de la Constitución Política de 1980, en términos de garantizar la protección del derecho a educación. En este sentido operarían los incrementos presupuestarios y el conjunto de medidas adoptadas en términos de infraestructura, equipamientos y estructura curricular, todo lo cual implicaría un enorme esfuerzo para generar el acuerdo social necesario (CORVALÁN, 2013: 77).

Cox (1997) resumía el ideario de los cambios señalando que ellos implicaban transitar desde un Estado "remero" a un Estado "timón", vale decir, de un Estado cuyo rol reside en ser no más que una parte del proceso de transformación a ser quien lo conduce estratégicamente ${ }^{5}$. Tal resumen, por otra parte, presenta el tono optimista y autocomplaciente de las reformas impulsadas por la Concertación hasta antes de 2006. Pues bien, en este escenario justamente poco a poco comenzarían a emerger partes del cuerpo político, manifestando su incomodidad, su desacuerdo y, en fin, su rechazo frontal a las políticas que orientaron el derrotero de la educación en los años señalados. Las manifestaciones buscarían subrayar que la realidad del sistema educativo chileno se asemejaba más a la regulación burocrático-administrativa, la ausencia de políticas y la evaluación como amenaza o riesgo, que a lo previsto como consecuencia de la acción del "timón estatal".

De este modo, al fragor de las movilizaciones estudiantiles del año 2006, se rompe lo que había sido hasta entonces una situación de pleno acuerdo en educación, y como reacción a ello, la Presidente Michelle Bachelet crea el Consejo Asesor Presidencial para la Calidad de la Educación ${ }^{6}$. El Consejo elaboró un Informe (2006) conteniendo un conjunto de recomendaciones que bien pueden contar como manifestación de lo que Burton llama la "etapa deliberativa" de la política educativa nacional, en la medida que un número importante de representantes de diversos sectores políticos y sociales participaron en la instancia generada a instancias del Poder Ejecutivo y en la cual se discutieron prácticamente la totalidad de las reivindicaciones propuestas en la opinión pública con la finalidad de establecer un acuerdo sobre su pertinencia y viabilidad.

Es en el Consejo Asesor del año 2006, donde se encuentran las distintas narrativas en torno al problema educativo mencionadas por Corvalán (2013): la centro-estatista, la de mercado y la síntesis de ambas, encarnada por las políticas de la Concertación. Ahí se discuten los elementos de continuidad -la educación como instrumento de igualdad en sociedades desiguales, la responsabilidad del Estado en proveer acceso a la educación y por los contenidos educativos-y también los de cambio, o que representan rupturas e innovaciones, tales como la autolimitación de la acción estatal como proveedora, la cesión de atribuciones a actores privados y la posibilidad de lucrar.

5 Es sugerente que la metáfora -pasar del "Estado remero" al "Estado timón"- provenga de un libro que lleva por título: "La reinvención del gobierno. La influencia del sector empresarial en el sector público" (OSBORNE y GAEBLER, 2002), puesto que ilustra la tendencia general del gobierno a reforzar el paradigma de lo privado por sobre lo público.

6 El Consejo Asesor contó con la participación de más de 80 personas que asistieron representado a: universidades y centros de estudio, estudiantes secundarios y universitarios, apoderados y profesores, representantes de las iglesias, funcionarios de la educación, de los pueblos indígenas, empresarios, fundaciones educacionales, legisladores, alcaldes, etc. 
Así también se discute sobre transformaciones de gran escala, tales como la redefinición de la relación entre Estado y Sociedad respecto a la provisión educativa, mediante el paso de una institucionalidad radicada en el Estado central a los mecanismos de mercado, y el paso de lo fiscal a lo municipal (CORVALÁN, 2013).

El hito constituido por el Consejo Asesor Presidencial para la Calidad de la Educación, da inicio a lo que Burton designa como de "representatividad restringida" (2007 a la fecha), que es la expresión manifiesta de las tensiones latentes, que no habían sido reconocidas ni intentado ser resueltas antes del mencionado Consejo, durante la "democracia de los acuerdos", que neutralizó las tensiones existentes y mantuvo la estabilidad política y social.

Unas tensiones que hoy se manifiestan, con nuevos bríos, dividiendo a la Alianza, a la Concertación y a la diversidad de grupos representados por lo que convendremos en llamar "Bloque Social", a partir de tres ámbitos que han sido frecuente fuente de conflicto en la sociedad: el lucro en la educación, la (re)definición del rol del Estado y la selectividad/segregación al interior del sistema educativo. Las diferencias de visión entre estos tres grupos, pueden consignarse de forma general según como las presenta la Tabla $N^{\circ} 1$ :

Tabla $N^{\circ}$ 1. Diferencias de visión entre distintos referentes políticos

\begin{tabular}{|c|c|c|c|}
\hline Dimensiones & Bloque Social & Concertación & Alianza \\
\hline Lucro & Opuesto a lucro & Diferentes posturas frente al \\
lucro & $\begin{array}{c}\text { Defiende el lucro } \\
\text { municipalizado }\end{array}$ & $\begin{array}{c}\text { Estado más débil, sistema } \\
\text { municipalizado y autonomía de } \\
\text { colegios }\end{array}$ \\
\hline Estado & $\begin{array}{c}\text { Estado central más fuerte y } \\
\text { menos municipalizado }\end{array}$ & $\begin{array}{c}\text { Más estado y mantener sistema } \\
\text { Regula selección }\end{array}$ & Defiende selección \\
\hline
\end{tabular}

Fuente: Adaptado de Bitar (2011), Burton (2012) y Picazo (2010)

El punto de la Tabla precedente es que ella refleja el consenso roto. Además, nótese que ninguno de los puntos centrales identificados por el Consejo Asesor Presidencial de 2006 fue recogido como contenido de algún proyecto de ley, pese a la amplia agenda legislativa existente en educación?

Como lo consigna la Tabla, el centro del debate corresponde al lucro, al rol del Estado y a la selectividad en el sistema. Entretanto, los distintos actores desde los profesores, al gobierno, pasando por el ministerio de educación, los legisladores, padres y apoderados, sostenedores y comunidad educativa (BITAR, 2011), presentan una "agenda propia", que es una mezcla entre reivindicaciones particularistas y deudas históricas.

Este es un escenario nuevo frente a la estabilidad ofrecida por un clivaje de largo aliento, en el que se debatían las distintas narrativas y actores en la educación que daban cuerpo al consenso descrito: (i) MINEDUCConcertación, (ii) Políticos de centro-derecha, Centro Estudios Públicos, Libertad y Desarrollo y El Mercurio. (iii) Izquierda extra-parlamentaria y Colegio de Profesores, (iv) Diferentes visiones de la evaluación del modelo y (v) Opinión pública y padres (PICAZO, 2010)

Los gobiernos de la Concertación, aprovechando este amplio consenso inicial apuntaron en sus políticas sociales a ciertos objetivos claves -reducción de la pobreza y mayor protección social focalizada-, pero estimaron

7 La discusión legislativa en educación aborda actualmente temas tales como: los deberes y derechos de los sostenedores, un nuevo sistema de aseguramiento de la calidad, reformas en el currículo, los mecanismos de financiamiento, la gestión escolar y los requisitos de los sostenedores y la creación del consejo nacional de educación, la desmunicipalización o, recientemente, la obligatoriedad de la enseñanza pre-escolar. 
que no era necesario alterar el marco heredado y la provisión privada, con fines de lucro, de la educación (SOLIMANO, 2012).

En este sentido, con el tiempo los avances en cobertura surgen más como una paradoja que como un logro, por ello, el hecho de que 7 de cada 10 jóvenes accedieran a la educación superior, como se dijo profusamente durante el gobierno del presidente Lagos, no debe leerse como un indicador de justicia social o como el ingreso definitivo a la sociedad del conocimiento, sino como las primeras luces de alerta respecto al lucro en la provisión de educación. En palabras de Solimano, corresponde hacerse cargo de la paradoja que surge al intentar: "cuadrar esta construcción hipotética de una aséptica y armoniosa economía de mercado con la evidencia diaria y tangible del capitalismo real" (2012:68).

La configuración de estos logros-como-paradojas posibilita una relectura de las políticas adoptadas durante los gobiernos de la Concertación, esto es, como "un fenómeno de reinterpretación de herencias institucionales autoritarias y de emergencia de nuevas ideas" (PICAZO, 2010:65). En este contexto, más que un mercado con oferta y demanda, pareciera que el sistema educativo se divide en ganadores y perdedores, en un juego en que pierden los públicos y quienes no pueden escoger (JOIKO, 2012) y en el que ganan los establecimientos particular subvencionados que pueden escoger y/o cobrar y los padres que pueden pagar y, por ende, escoger.

Mirando al futuro inmediato, la pregunta es si todo este proceso de subjetivación, esta integración de "los sin parte" que han logrado irrumpir en el espacio hegemónico que se había configurado entre los actores tradicionales y los policy makers, logrará transformar el ruido en lenguaje: la protesta callejera en acción institucional. De ser ello posible, entonces habrá que esperar si será posible entonces neutralizar las discrepancias manifiestas y generar políticas públicas que ofrezcan soluciones efectivas a los problemas del presente. O si, por el contrario, se seguirán presentando propuestas y soluciones, que luego se convertirán en paradojas, en la medida que no atacan los problemas centrales -lucro, rol del Estado o la segregación escolar, por ejemplo- que han sido sistemáticamente denunciados desde las movilizaciones estudiantiles los años 2006 y 2011.

La discusión de la sociedad chilena en torno al lucro con bienes públicos, que también se extiende a ámbitos como la salud o las pensiones, por lo pronto implica una redefinición del rol que debe jugar el Estado en su regulación y provisión. De ahí que el nuevo contexto político y social plantee una situación de incertidumbre respecto a la construcción de consensos, en que existen nuevos actores, y se han visto fortalecidos y debilitados los antiguos.

El problema, ya sea situado en su diseño institucional, el financiamiento y la regulación los mecanismos de mercado, es que las soluciones propuestas hasta ahora no guardan relación con las preguntas planteadas, y la "agenda negada", aquella sobre que la OCDE advirtiera en 2004 y el movimiento de los estudiantes secundarios impusiera en 2006 (COX, 2012), sobre la que se sigue discutiendo hoy y seguiremos discutiendo los próximos años, no ha logrado ser puesta en el debate efectivo.

\section{Consideraciones finales}

A fines de los años 70, J. F. Lyotard (1987) se preguntaba en relación con la situación del saber, de la economía y de la política en Québec, Canadá: “¿Quién decide lo que hay que saber? ¿Quién sabe lo que hay que decidir?", planteándose premonitoriamente la cuestión central de la transición a la democracia chilena así como la cuestión central de la política educativa nacional: mientras que en el primer caso señalaba a la legitimidad política, en el otro planteaba la cuestión del estatus del saber.

En parte, ello se expresa en la complejas relaciones que mantiene la educación con el desarrollo y con los derechos humanos y en el reconocimiento de la triple dimensión del desarrollo a que la educación contribuye, a saber, a generar condiciones de equidad, elevar la productividad y mejorar las bases de la experiencia de ciudadanía (OTTONE y HOPPENHAYN, 2007). 
Estos efectos de una "buena" educación han sido lastimosamente reducidos a su dimensión puramente económica, bajo la forma de capital humano, limitando así el amplio valor de la educación en el florecimiento humano. En efecto, en tanto se ha pensado que la educación es una palanca del desarrollo económico, tiende a diluirse la exigencia que deriva de la condición de derecho de la educación. Por el contrario, cuando la educación se defiende como un derecho, tiende a perder valor la idea que ésta sea una parte fundamental del desarrollo. En Chile esta tensión es la que ha estado a la base de la discusión educacional. Es la tensión que ha originado las mayores dificultades para el proceso político, por cuanto suscita la pregunta acerca de ¿Qué propósito tiene la educación? ¿A quién le corresponde su provisión? ¿Cuál es la fuente de su financiamiento? ¿Cuán selectivo debe ser el sistema educacional?

Durante años la educación sería comprendida como un bien de apropiación privada, un bien cuyo acceso reporta beneficios individuales o privados. Bajo esta premisa se justificó sin límite alguno la idea de que su costo, el costo de educarse, debía ser asumido por quienes habrían de beneficiarse de la inversión, vale decir, los propios estudiantes y sus familias. La preeminencia de esta idea produciría la retirada del Estado: sin asegurar acceso ni cobertura educativa, el Estado navegaba por así decir entre Scila y Caribdis, entre la figura del Estado "remero" y la del "timón".

El sistema educacional chileno transformó la justificada y razonable aspiración de los padres en una maquinaria de desigualdad social y la sociedad chilena tardaría en alcanzar consciencia del efecto socialmente devastador de las decisiones que estaban efectuándose en el terreno de la política educacional, en orden a someter el funcionamiento de la educación al régimen del mercado.

En parte, la limitación de la perspectiva sobre la educación se debía precisamente a la creencia de estar a cubierto del caos por el consenso político. El consenso fue entendido así como en antídoto contra la amenaza del caos social. El consenso cubría las grietas de la institucionalidad política allí donde dicha institucionalidad no bastaba para cerrar del todo las múltiples brechas de un pacto en el cual se había logrado enquistar la desigualdad y para el cual, de hecho, la desigualdad era una condición de posibilidad.

Poner bajo control la situación consistió en la atribución básica de la transición, una situación histórica que revelaba bien de dónde se había partido pero cuyo destino era, con suerte, incierto. En verdad, sin embargo, se trató del relato de un extravío democrático que sirvió como la abreviatura de un trazado que Ilevaría a Chile de la Dictadura a la Postpolítica, sin solución de continuidad. La transición política chilena no fue en sí misma un momento democrático, en parte importante porque su dinámica interna exigía que no fuera siquiera un "momento".

Así como se llamó a la Dictadura "paréntesis en la vida republicana" -una epojé de 20 años por hablar solo de sus efectos visibles-, la transición a la democracia sería una "suspensión": la ciudadanía permanecía ausente mientras tanto la situación se normalizaba, si la metáfora se interpreta temporalmente; el país estuvo suspendido, colgando del metaestable equilibrio aportado por el consenso de los que tienen parte, si se interpreta espacialmente.

Sin embargo, lo que llegaría a ser normal fue justamente la transición misma porque la transición nos acostumbró a la falta, al duelo. A hablar bajito para que no se oyera el lenguaje detrás del ruido, el sentido por sobre el rumor: Chile se encontraba sumergido en aguas turbias y de esas aguas no lograría salir inmaculado. Hoy, con idénticas preguntas a aquellas que se formulara el año 2006 en el Consejo Asesor, aún no hay respuestas sencillas, ni que generen un consenso o la aceptación de todos los actores, la construcción de ese consenso y la construcción de un nuevo clivaje que sea capaz de dar respuesta a estas preguntas será probablemente lo que nos ocupe durante los próximos años. 


\section{Referencias Bibliográficas}

ALMONACID, C. Un cuasi mercado educacional: la escuela privada subvencionada en Chile. Revista de Educación. Número 333, pp. 165-196. 2004.

ALMONACID, C. La educación particular subvencionada como cooperadora del Estado. En: La agenda pendiente en Educación. Profesores, administradores y recursos: propuestas para la nueva arquitectura de la educación chilena. Editores: Bellei, Cristián; Contreras, Daniel; Valenzuela, Juan Pablo. Universidad de ChileUNICEF. 2008

ATRIA, F. Mercado y ciudadanía en la educación. Flandes Indiano. Santiago. 2007.

BELLEI, C.; CONTRERAS, D. y VALENZUELA, J.P. Viejos dilemas y nuevas propuestas en la política educacional chilena. En: Ecos de la revolución ping üina. Avances, debates y silencios de la reforma educacional. Editores: Bellei, C., Contreras, D.; Valenzuela, J.P. Universidad de Chile-UNICEF. Pp 11-27. 2010.

BITAR, S. Cómo construir consensos en educación. PREAL. Programa de Promocón de la reforma educativa en América Latina y el Caribe. Agosto, 2012.

BURTON, G. Hegemony and Frustration: Education Policy Making in Chile under the Concertación 19902010. Latin American Perspectives. 2012 39:34. 2012.

Consejo Asesor Presidencial para la Calidad de la Educación. Informe final de consejo asesor presidencial para la calidad de la educación. 2006. Disponible en http://mt.educarchile.cl/MT/jjbrunner/archives/libros/ ConsejoAsesor/Inf_def.pdf (Acceso: 11/03/2013).

CORVALÁN, J. La narrativa educacional chilena y su proceso de transformación reciente: un análisis sociológico-histórico. Folios Segunda Época 2013 pp. 63 -81 Universidad Pedagógica Nacional Facultad Humanidades.

COX, C. La reforma de la educación chilena: contexto, contenidos, implementación. En Documento de trabajo No 8. Programa de Promoción para la Reforma Educativa en América Latina y el Caribe - PREAL. 1997.

COX, C. Política y Políticas educacionales en Chile 1990-2010. Revista Uruguaya de Ciencia Política. Vol. 21 No1 ICP Montevideo, pp. 13-42. 2012.

FUKUYAMA, F. El fin de la historia y el último hombre. Editorial Planeta, Barcelona. 1993.

GARRETÓN, M.A.; GARRETÓN, R. La transición incompleta en Chile: la realidad tras los rankings internacionales. Revista de Ciencia Política, v. 30, n. 1, p.115-148, 2010.

GODOY, O. La Transición Chilena a la Democracia: Pactada. Estudios Públicos, 74 (otoño 1999).

HOPENHAYN, M. Ni apocalípticos ni integrados: aventuras de la modernidad en América Latina. Fondo de Cultura Económica, Santiago, 1994.

JOIKO, S. El cuasi-mercado educativo en Chile: Desarrollo y consecuencias. Revista electrónica Diálogos educativos. Vol. 12 año 2012.

KRIPPNER, G. y ALVAREZ, A. Embeddedness and the Intellectual Projects of Economic Sociology. Vol.33, p.219-240. 2007.

LIPOVETSKY, G. El Crepúsculo del Deber. La ética indolora de los nuevos tiempos. Barcelona: Anagrama. 1993. LYOTARD, J.F. La condición postmoderna. Informe sobre el saber. Ed. Cátedra. 1987.

MIZALA, A. y ROMAGUERA, P. Calidad de la educación chilena: el desafío de la próxima década. En: La paradoja aparente. Equidad y eficiencia: resolviendo e dilema. Taurus. 2005

MOULIAN, T. Chile actual: anatomía de un mito. Santiago: LOM ediciones. 1997.

OSBORNE, D. y GAEBLER, T. La Reinvención del Gobierno. La influencia del esp íritu empresarial en el sector público. Ediciones Paidós Iberica, Barcelona. 2002.

OTTONE, E. y HOPENHAYN, M. Desafíos educativos en la sociedad del conocimiento. Pensamiento educativo. Vol. 40. No1, pp. 13-29. 2007. 
Panel de Expertos para una Educación de Calidad. Informe final. Segunda etapa: Fortalecimiento de la institucionalidad pública. 2011. Disponible en: http://www.mineduc.cl/usuarios/mineduc/doc/201103291620330. INFORMEFINALPANELDEEXPERTOS1.pdf. Acceso: 06.03.2013.

PICAZO, I. La metamorfosis de la regulación pública en la educación escolar en Chile: hacia un estado postneoliberal. Revista Pensamiento Educativo. Vols.46-47, 2010 pp63-91. 2010.

RANCIĖRE, J. El desacuerdo. Política y filosofía. Buenos Aires: Nueva Visión. 1996.

RANCIĖRE, J. Sobre políticas estéticas. Barcelona: Museo de Arte Contemporáneo. 2005.

RICHARD, N. La Insubordinación de los signos: cambio político, transformaciones culturales y poéticas de la crisis. Santiago de Chile: Cuarto Propio. 1994.

SOLIMANO, A. Capitalismo a la chilena y la prosperidad de las elites. Santiago: Editorial Catalonia. 2012.

ŽIŽEK, S. En defensa de la intolerancia. Ed. Sequitur, Madrid. 2008.

Enviado e Aprovado em outubro de 2013 